OPEN ACCESS

Edited by:

Uday Kishore,

Brunel University London,

United Kingdom

Reviewed by:

Sreya Ghosh,

Boston Children's Hospital and Harvard Medical School, United States

Abhishek Shastri,

Central and North West London NHS

Foundation Trust, United Kingdom

*Correspondence:

Hua Bai

baih2020@gmc.edu.cn

Specialty section:

This article was submitted to

Molecular Innate Immunity,

a section of the journal

Frontiers in Immunology

Received: 28 April 2021

Accepted: 12 July 2021

Published: 26 July 2021

Citation

Bai H and Zhang Q (2021) Activation of NLRP3 Inflammasome and Onset of Alzheimer's Disease.

Front. Immunol. 12:701282. doi: 10.3389/fimmu.2021.701282

\section{Activation of NLRP3 Inflammasome and Onset of Alzheimer's Disease}

\author{
Hua Bai ${ }^{1,2,3 *}$ and Qifang Zhang ${ }^{4,5}$ \\ ${ }^{1}$ Department of Neurology, The Third Affiliated Hospital of Guizhou Medical University, Duyun, China, ${ }^{2}$ Department of \\ Neurology, Affiliated Hospital of Guizhou Medical University, Guiyang, China, ${ }^{3}$ Medical Experimental Center of the Third \\ Affiliated Hospital of Guizhou Medical University, Duyun, China, ${ }^{4}$ Key Laboratory of Endemic and Ethnic Diseases, Ministry of \\ Education, Guizhou Medical University, Guiyang, China, ${ }^{5}$ Key Laboratory of Medical Molecular Biology, Guizhou Medical \\ University, Guiyang, China
}

The nucleotide-binding domain leucine-rich repeat and pyrin domain containing receptor protein 3 (NLRP3) is an important pattern recognition receptor in human innate immunity. Activation of the NLRP3 inflammasome play a key role in the pathogenesis of Alzheimer's disease (AD). Theories explaining activation of the NLRP3 inflammasome include the reactive oxygen species theory, the lysosomal damage theory and the mitochondrial DNA theory. The NLRP3 activation promotes occurrence of AD by producing IL-1 $1 \beta, \mathrm{IL}-18$ and other cytokines, and then by affecting the deposition of $A \beta$ and tau proteins. Overactivated NLRP3 inflammasome often impair cell function and induces immune-related diseases. Some mechanisms have been found to negatively regulate activation of the NLRP3 inflammasome, which may be through receptor binding blocking mechanism, autophagy related mechanism, abnormal cytokine secretion mechanism, or interference related gene expression regulation mechanism. In this review, we summarize the possible mechanisms by which the activation of NLRP3 inflammasomes affects the pathogenesis of $A D$, and the recent advances in the prevention and treatment of $A D$ by controlling the activation of NLRP3 inflammasomes. By researching the activation or inactivation of NLRP3 inflammasome, it is possible to reveal the pathogenesis of AD from a new perspective and provide a new idea for the prevention and treatment of $A D$.

Keywords: Alzheimer's disease, inflammasome, pathogenesis, activation, nucleotide-binding domain leucine-rich repeat and pyrin domain containing receptor protein 3

\footnotetext{
Abbreviations: A $\beta$, $\beta$-amyloid protein; ABRO1, Abraxas Brother 1; AD, Alzheimer's disease; APP, Amyloid precursor protein ASC, Apoptosis associated speck like protein containing cysteine aspartic proteinase recruitment domain; ATP, Adenosine triphosphate; BRCC3, Breast cancer susceptibility gene complex subunit protein 3; CARD, Cysteine aspartic proteinase recruitment domain; CD36, Cluster Of Differentiation 36; EPM, Endogenous pathogenic molecules; GMF, Glial mature factor; D4T, 2',3'-Didehydro-3'-deoxythymidine (Stavudine); DAMP, danger associated molecule patterns; IL-18, Interleukin 18; LPS, Lipopolysaccharide; LTP, Long term potentiation; MG132, Carbobenzoxy-Leu-Leu-leucinal; mtDNA, Mitochondrial

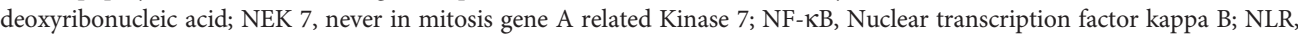
Nucleotide-conjugated oligomeric domain-like receptor; NLRC4, Nucleotide-conjugated oligomeric domain-like receptor family cysteine aspartic proteinase recruitment domain domain-containing 4; NLRP3:Nucleotide-binding domain leucine-rich repeat and pyrin domain containing receptor protein 3; NOD, Nucleotide-conjugated oligomeric domain; P2X7R, Purinergic ligand-gated ion channel 7 receptor; PAMP, Pathogen-associated molecular pattern; PG, Progesterone; pro-IL-1 $\beta$, Interleukin1 beta precursor protein; PS1, Presenilin-1; PYD, pyrimidine nucleoside; ROS, Reactive oxygen species; RS, Radix Scrophulariae; STAT, Signal transduction and transcriptional activation factor; TRX, Thioredoxin; TXNIP, Thioredoxin interaction protein.
} 


\section{INTRODUCTION}

With the increase in the elderly population, the incidence of Alzheimer's disease (AD) has a trend of increasing year by year (1). $\mathrm{AD}$ is a neurodegenerative disease. Its main clinical manifestations are memory disorder, personality abnormality, apraxia, visual space abnormality, executive dysfunction and neuropsychiatric symptoms $(2,3)$. The pathogenesis of $\mathrm{AD}$ is mainly related to the deposition of $\beta$-amyloid protein $(A \beta)$, the neurofibrillary tangle caused by phosphorylation of microtubule associated protein and the loss of neurons. Compared with the healthy brain, the brains of $\mathrm{AD}$ patients have a significant accumulation of tau protein and interacts with abnormal $A \beta$ proteins (4). The main pathological changes in the brain of $\mathrm{AD}$ patients under the microscope are senile plaques, neurofibrillary tangles, vacuolar degeneration of neuronal particles, cerebral amyloid angiopathy and glial cell hyperplasia (3). The above pathological changes are closely related to the innate immune abnormalities in the brain, in which the non-specific damage of brain blood vessels and the immune inflammatory response of glial cells play important roles $(5,6)$.

The etiology of $\mathrm{AD}$ are various and hidden, and the pathogenesis of $\mathrm{AD}$ is complex and varied. However, the role of inflammasomes, especially the nucleotide-binding domain leucine-rich repeat and pyrin domain containing receptor protein 3 (NLRP3) inflammasome, has recently been highlighted (7-9). NLRP3 is a very important pattern recognition receptor in human innate immunity. As an indispensable component of natural immunity, the NLRP3 inflammasome plays a key role in the human immune response and onset of some immune-related diseases (10). The NLRP3 inflammasome can regulate activation of caspase-1, thereby promoting maturation and secretion of the cytokines interleukin-1 beta precursor protein (pro-IL-1 $\beta$ ) and pro-IL-18 during immune defense (11). More and more experimental evidence show that the activation of NLRP3 inflammasome is closely related to some immune related diseases, such as gout, multiple sclerosis, diabetes, ulcerative colitis and AD (12). Recently, great progress has been made in the study of NLRP3 inflammasome associated with $\mathrm{AD}$.

\section{COMPOSITION AND FUNCTION OF NLRP3 INFLAMMASOME}

The inflammasome is composed of molecular receptor protein, adapter protein and pro-caspase-1. Molecular receptor proteins include classical NLR and non-classical NLR. Adapter protein here is an apoptosis-associated speck-like protein containing cysteine aspartic proteinase recruitment domain (ASC), Generally speaking, the adapter protein is ASC. ASC is a cohesive protein of this specific inflammasome, whose amino terminal contains pyrin domain (PYD) domain and carboxyl terminal contains cysteine aspartic proteinase recruitment domain (CARD) domain. The CARD domain acts as a recruitment effector protein, which can be matched and linked with the CARD domain in the caspase-1 precursor protein (13). Different inflammasomes are formed by different molecular receptor proteins. Some inflammasomes do not require ASC $(14,15)$. Inflammasomes are a kind of multi-protein complex assembled by intracytoplasmic pattern recognition receptors. They recognize the foreign pathogen molecules and the host's own danger signals, to recruit and activate the proinflammatory protease pro-caspase-1. Activated caspase- 1 can enzymatically digest and chop the precursors of IL- $1 \beta$ and IL-18 to produce the corresponding mature cytokines (16). At present, it is certain that a variety of inflammasomes participate in the host's defense response against pathogens, and pathogens have also evolved multiple mechanisms to inhibit the activation of inflammasome (17).

The NLRP3 inflammasome is a complex composed of NLRP3, ASC and caspase-1 precursor proteins, in which NLRP3 protein plays a leading role (18). NLRP3 is a NOD like receptor protein with pattern recognition, with carboxyl terminus and leucine rich repeat domain, but lacking CARD domain. So it is not possible to bind to caspase-1 precursor protein directly. Instead, it is necessary to recruit caspase-1 precursor protein by binding to ASC through the pyrin domain at the $\mathrm{N}$-terminal, and then activate caspase-1 (Figure 1). NLRP3 binds the adaptor ASC through PYD-PYD interactions, and ASC binds pro-caspase-1 via CARD-CARD domain interactions. The caspase- 1 precursor protein first forms a tetramer for self-activation, followed by a heterodimer with enzymatic activity (19). Caspase-1, as an inflammasome effector protein, is able to cut the inactive IL- $1 \beta$ precursor protein and IL18 precursor protein into mature IL-1 $\beta$ and IL- 18 , respectively, so as to play a variety of non-specific inflammatory roles (20). The abnormality of NLRP3 inflammasome is closely related to the onset of various refractory diseases (such as diabetes, $\mathrm{AD}$, etc.) (21). Therefore, it is of great clinical significance to study the activation and function of the NLRP3 inflammasome.

\section{ACTIVATION OF NLRP3 INFLAMMASOME}

The initiation signals of activating NLRP3 inflammasome are some internal and external activators, including a variety of pathogen-associated molecular patterns (PAMP) and danger associated molecule patterns (DAMP). The PAMP signals that can be detected by the NLRP3 inflammasome are silica, calcium pyrophosphate, sodium urate, palmitate, cholesterol, sodium A, and certain viruses. Lipopolysaccharide (LPS) is often considered a prototypic PAMP. Except for viruses, other pathogenic factors mentioned above are sometimes considered to belong to DAMP (22). It has been found that DAMP signals that can be sensed by the NLRP3 inflammasome include hypokalemia, hypercalcemia, hyperglycemia, cathepsin B, adenosine triphosphate (ATP), and reactive oxygen species (ROS), etc. (Table $\mathbf{1}$ ).

Activation of the NLRP3 inflammasome by foreign signals involves complex pathophysiological and biochemical processes, and currently there are mainly three theories to explain this activation mechanism (23). The first theory is the reactive oxygen theory, which believes that reactive oxygen species (ROS) are key 


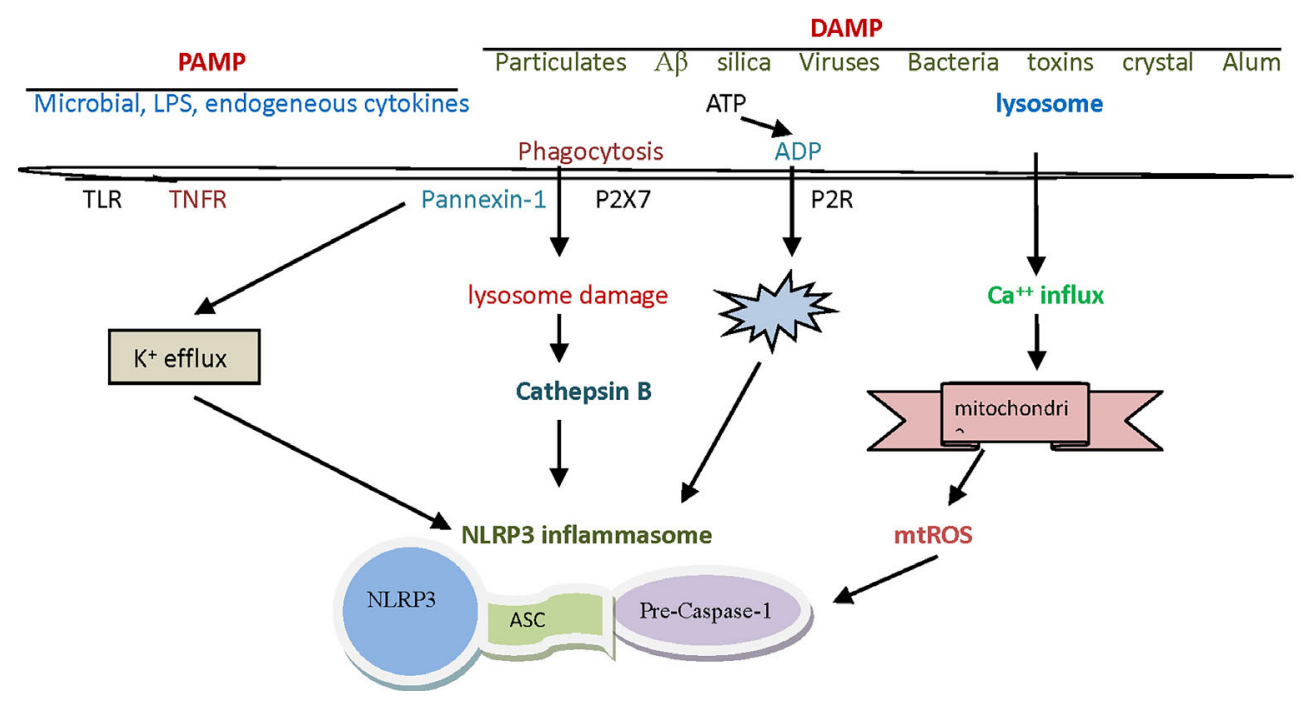

FIGURE 1 | The assembly and activation of The NLRP3 inflammasome. The NLRP3 inflammasome is a complex composed of NLRP3, ASC and caspase-1 precursor proteins, in which NLRP3 protein plays a leading role. ASC is a cohesive protein of this specific inflammasome, whose amino terminal contains pyrin domain and carboxyl terminal contains CARD domain. The initiation signals of activating NLRP3 inflammasome are some internal and external activators, including a variety of PAMP and DAMP. NLRP3, nucleotide-binding domain leucine-rich repeat and pyrin domain containing receptor protein 3; ASC, apoptosis associated speck like protein containing cysteine aspartic proteinase recruitment domain; CARD, cysteine aspartic proteinase recruitment domain; PAMP, pathogen-associated molecular patterns; DAMP, danger associated molecule patterns.

mediators in the regulation of NLRP3 inflammasome, and that it is associated with high concentration or high expression of catalase or reduced nicotinamide adenine dinucleoside phosphate oxidase. In normal physiology, the thioredoxin interaction protein (TXNIP) and thioredoxin (TRX) are bound to each other. When certain oxidative cell stress increases ROS, TRX oxidizes itself in order to remove ROS, TXNIP and TRX are separated (18). TXNIP was then induced to bind to NLRP3, thereby relying on ASC to recruit caspase-1 precursor protein, and finally to complete the assembly and activation of the NLRP3 inflammasome (Figure 1). Crystals, high glucose, ATP and other activators (activation signals) usually activate NLRP3 inflammasome in this way $(24,25)$. Another theory is the lysosomal damage concept, which believes that PAMP, when swallowed by macrophages, destabilizes the phagocytes, leading to lysosomal acidification and rupture, from which cathepsin B is normally released into the cytoplasm. Since cathepsin B can degrade the inhibitory protein of NLRP3, it can activate the inflammasome of NLRP3 (26).

Macrophages must first be exposed to initiating stimuli to activate the transcription factor NF-kB through toll-like receptor ligands or cytokine receptors, to up-regulate NLRP3 expression.
Molecules that regulate NF-kB activity can indirectly affect NLRP3 priming. Activated NF-kB transcribes NLRP3 mRNA and then performs translation and post-translational modifications to complete the NLRP3 priming process (27). The third theory is the mitochondrial DNA theory, which believes that various PAMP or DAMP in the body can attack or damage the mitochondria in the body's cells under certain conditions. Damaged mitochondria often release mitochondrial deoxyribonucleic acid (mtDNA), which activates NLRP3 inflammasome by potassium outflow or calcium influx. In this activation mode, the destruction of cell membrane by cytotoxin, ATP binding to purinergic ligand-gated ion channel 7 receptor (P2X7R), and the intervention in mitochondrial micropore structure by microbial toxin may contribute to potassium ion outflow. Calcium influx may be assisted by G-protein-coupled receptor, calcium-sensitive receptor, and pannexin-1 channels $(28,29)$.

Activation of the NLRP3 inflammasome usually requires two steps: first, initiation of an inflammatory response, and second, oligomerization of NLRP3 and assembly with ASC and caspase-1 precursors (30). The inflammatory response is usually the result of uninhibition and nuclear transposition of the nuclear

TABLE 1 | The activated mode and steps of NLRP3 inflammasome and the theories that explain these activation.

\begin{tabular}{|c|c|c|c|}
\hline & Activating molecules & Activation steps & Theories for explaining these activation \\
\hline PAMP & $\begin{array}{l}\text { Calcium pyrophosphate, sodium, LPS, urate, } \\
\text { palmitate, cholesterol, etc. }\end{array}$ & $\begin{array}{l}\text { Inducing inflammation, oligomerization of NLRP3, assembly } \\
\text { of ASC and caspase-1 precursor protein. }\end{array}$ & $\begin{array}{l}\text { Active oxygen theory, lysosomal damage } \\
\text { theory, mitochondrial DNA theory }\end{array}$ \\
\hline DAMP & $\begin{array}{l}\text { Hypokalemia, hypercalcemia, cathepsin B, Silica, } \\
\text { A } \beta \text {, ATP, hyperglycemia, ROS, etc. }\end{array}$ & $\begin{array}{l}\text { oligomerization of NLRP3, assembly of ASC and caspase-1 } \\
\text { precursor protein. }\end{array}$ & $\begin{array}{l}\text { Active oxygen theory, mitochondrial DNA } \\
\text { theory }\end{array}$ \\
\hline
\end{tabular}


transcription factor kappa $\mathrm{B}(\mathrm{NF}-\kappa \mathrm{B})$, which leads to transcription of NLRP3 itself and IL-1 $\beta$. Many hormoneinduced signaling pathways are reduced by the activation of NF- $\kappa B$. The stimulation of LPS signals through toll-like receptor 4/leukocyte differentiation antigen 14 (TLR4/CD14) signaling pathway. The deubiquitination of NLRP3 is thought to depend on the role of mitochondrial ROS. The mitotic kinase NEK7 mediates the assembly and activation of NLRP3 inflammasome during cell division. NEK7 connects adjacent NLRP3 subunits and mediates the activation of NLRP3 inflammasome by interaction (31). Sharif et al. (32) reported that structural identification of NLRP3 and NEK7 was confirmed by in vitro and intracellular mutations. NEK7 mediates the activation of NLRP3 inflammasome by interacting with the two parts and connecting adjacent NLRP3 subunits. Ren et al. found (33) that ABRO1 promoted the activation of NLRP3 inflammasome by regulating NLRP3 dihydroylation. ABRO1 as a scaffold for the interaction between NLRP3 and breast cancer susceptibility gene complex subunit protein 3 (BRCC3), acting synergistically with BRCC3 promotes the inflammatory activation of NLRP3 by regulating the deamination of NLRP3. In addition, blocking the activity of ABRO1 can prevent the activation of the relevant inflammatory cells.

Changes in potassium and calcium ion concentrations can affect activation of the NLRP3 inflammasome. The classical NLRP3 activation pathway can lead to the increase of intracellular calcium ion concentration, enhancing the intracellular calpain activity. When membrane potential is depolarized by high concentration of potassium ions in extracellular solution, or is hyperpolarized by small molecule compounds, calpain activity can be decreased and activation of
NLRP3 inflammasome can be blocked (13). Thus, it was confirmed that the continuous changes of membrane potential inhibited the activation of calpain, which was thought as a new molecular mechanism of calpain regulating the assembly of NLRP3 inflammasome under resting membrane potential (34). In addition, the treatment of high concentration of potassium ions outside the cell caused the continuous depolarization of cell membrane potential by obstructing the outflow of potassium ions, which have dramatic impact on intracellular signal transduction and activity of biological macromolecules. Although NLRP3 inflammasome can be activated during the early stages of crystal stimulation without decreasing intracellular potassium concentration, sometimes the initiation of NLRP3 inflammasome activation signaling is not affected by the concentration of potassium efflux (35).

\section{NEGATIVE REGULATION OF NLRP3 INFLAMMASOME ACTIVATION}

Proper activation of NLRP3 inflammasome may be beneficial to the human body under certain conditions as it can help to resist exogenous microbial infection and endogenous cell damage. Overactivation of the inflammasome, however, is usually detrimental to cellular and body health. At present, it has also been found (36) that there are various mechanisms in cells of the body for the negative regulation of the activation of NLRP3 inflammasome, which may function through the mechanism of receptor binding and blocking, the mechanism of autophagy, or by interfering with the expression mechanism of related genes (Figure 2). For example, mir-223 negatively regulates the

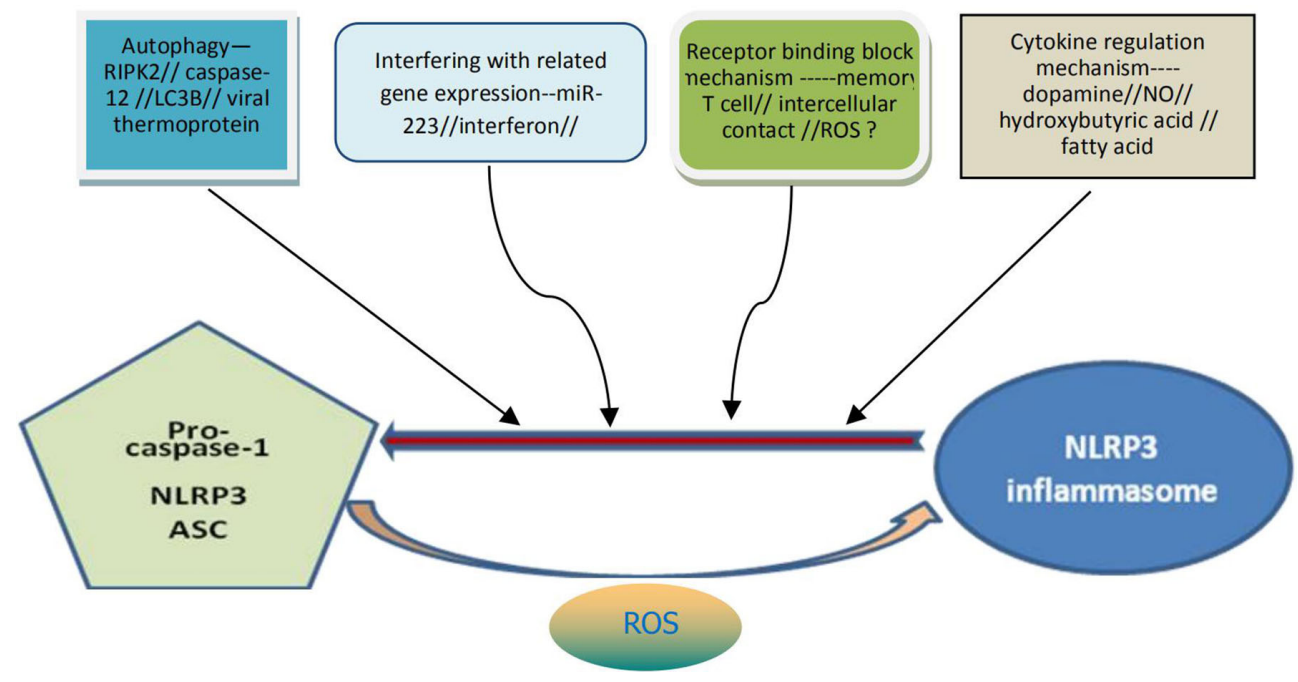

FIGURE 2 | Possible specific mechanisms for negative regulation of NLRP3 inflammasome activation. The activation of NLRP3 inflammasome can be negatively regulated through at least four pathways: the mechanism of autophagy, the mechanism by interfering with the related genes expression, the mechanism of receptor binding and blocking, the mechanism from cytokine regulation. It can also be understood that the above four pathways contribute to the decomposition and destruction of NLRP3 inflammasome. ROS may be dual and generally contribute to the formation and activation of NLRP3 inflammasome. NLRP3, nucleotidebinding domain leucine-rich repeat and pyrin domain containing receptor protein 3; ROS, reactive oxygen species. 
activation of NLRP3 inflammasomes by inhibiting the expression of NLRP3 gene at the transcriptional level. T cells and interferon inhibit activation of the NLRP3 inflammasomes by down-regulating the expression of P2X7R and interfering with signal transduction and transcriptional activation factor (STAT) signaling pathways, respectively. Bai et al. (37) found that hydrogen peroxide treatment activated NLRP3 inflammasome and reduced signal transduction and phosphorylation of STAT serine 727 in vitro cultured cells. Preconditioning with BAPTA-AM inhibited the activation of NLRP3 inflammasome induced by hydrogen peroxide. Downregulation of STAT gene expression may enhance NLRP3mediated oxidative stress, a process independent of calcium signaling. In addition, dopamine, nitric oxide, $\beta$-hydroxybutyric acid and unsaturated fatty acids can inhibit activation of the NLRP3 inflammasome. The increase of autophagy protein LC3B will lead to the decrease of ROS level, thus inhibiting activation of the NLRP3 inflammasome (Figure 1). Viral thermal proteins can interfere with the binding between ASC and NLRP3 by PYD-PYD, thus negatively regulating the assembly and activation of the NLRP3 inflammasome. Caspase-12 also negatively regulates activation of the NLRP3 inflammasome through CARD-CARD competitively binding ASC (38).

Mitochondria play an unique role in the activation and regulation of the NLRP3 inflammasome. Localization of NLRP3 in mitochondria is critical for activation of the NLRP3 inflammasomes, and mitochondrial adaptor proteins are necessary for optimal activation of the NLRP3 inflammasome. Mitochondrial antiviral signaling protein not only mediates the response of type I interferon against virus, but also as a bridge for mitochondrial regulation of the NLRP3 inflammasome (39). Actin-dependent mitochondrial ASC transfer is mediated by acetylation, which causes the mitochondrial ASC to interact with the endoplasmic reticulum NLRP3. Autophagy related proteins can maintain mitochondrial integrity and inhibit the NLRP3 inflammasome activation. During influenza virus infection, RIPK2 protein can inhibit the activation of NLRP3 inflammasome by promoting mitochondrial autophagy (40).

The regulatory effect of ROS on the NLRP3 inflammasome remains controversial. Although some experiments have proved that ROS can mediate activation of the NLRP3 inflammasome, others have found that ROS can only promote the activation of NLRP3 protein, but have no effect on the activation of the NLRP3 inflammasome (41). In the resting state, the expression level of NLRP3 in cells is very low, so the activated NF- $\kappa B$ function is needed to transcribed NLRP3 mRNA, and then the translation and post-translation modification can be carried out. In addition, lysosomes, as an organelle for the decomposition of various macromolecules in eukaryotic cells, also participate in the regulation of activation of the NLRP3 inflammasome. These phagocytic particles may promote the activation of NLRP3 inflammasome by acting directly with inflammasome related proteins, and may also produce some ROS, which play a negative feedback regulating role in the activation of NLRP3 (42).

There are also very unique ways in which NLRP3 inflammasome activation is negatively modulated. Type I interferon (IFN) inhibits the production of interleukin-1 and the activation of inflammasomes. IFN inhibits the production of IL-1 $\beta$ through two different mechanisms. The IFN signaling pathway inhibits the activity of NLRP1 and NLRP3 inflammasomes through the STAT1 transcription factor, thereby inhibiting caspase-1-dependent IL-1 $\beta$ maturation. In NLRP3 dependent peritonitis models, effector T cells reduced neutrophils recruitment in an antigen-dependent manner, demonstrating that $\mathrm{T}$ cells inhibit innate immune response by inhibiting NLRP3 inflammasomes (43).

\section{SOME CLUES TO THE ONSET OF AD AFTER THE ACTIVATION OF NLRP3 INFLAMMASOME}

When the NLRP3 inflammasome is activated, it can promote the onset of $\mathrm{AD}$ in two ways. First, it can regulate IL-1 $\beta$, which produces neurotoxins that cause degeneration of neurons. Second, it can cause an increase in $A \beta$ deposition, thus inducing $A \beta$ self-perpetuating positive feedback loop, which eventually leads to the development of $\mathrm{AD}$. The exposed complex of ASC with A $\beta$ can amplify proinflammatory response, lead to inflammatory cell death, release functional ASC and induce a vicious cycle of feedforward stimulation.

NLRP3 inflammasome is activated not only by fibrillar $A \beta$ aggregates, but also by lower molecular weight $A \beta$ oligomers and protofibrils (44). It has been found that high expression of NLRP3 and caspase- 1 genes was conducive to the formation of aging lesions in the brains of amyloid precursor protein/presenilin-1 (APP/PS-1) transgenic mice (45). By reducing the activity of caspase- 1 and IL$1 \beta$ in the brain, it help clear $A \beta$. By reducing the activity of caspase- 1 and IL- $1 \beta$ in the brain, it is helpful to eliminate A 3 . In NLRP3 knockout mice, $A \beta$ deposition was reduced and spatial memory was improved (9). On the other hand, the activation of the NLRP3 inflammasome can reduce the phagocytosis of microglia on $A \beta$, thus increasing $A \beta$ deposition and promoting the occurrence and development of $\mathrm{AD}$ lesions (46).

Using the autopsy brains of human $\mathrm{AD}$ patients and age matched non $\mathrm{AD}$ patients as the control materials, the relationship between the NLRP3 inflammasome and autophagy lysosome labeled A0205 protein, or p-tau protein, or glial maturation factor (GMF) was analyzed by immunohistochemistry, Ahmed et al. found (47) that the neuroinflammatory effect promoted by the NLRP3 inflammasome can be amplified and regulated by GMF, and further reduce the clearance of protein aggregates mediated by autophagy signaling pathway. Saresella et al. (48) found that in peripheral monocytes of patients diagnosed with $\mathrm{AD}$, there was evidence of activation of both the NLRP3 inflammasome and the NLRP1 inflammasome after LPS or A $\beta$ stimulation. They believed that the migration of peripheral monocytes in the blood-brain barrier was likely to be an important factor leading to $\mathrm{AD}$ neuroinflammation. Nucleoside reverse transcriptase inhibitors can inhibit activation of these inflammatory factors. Activation of the NLRP3 inflammasome can also promote the pathological formation of tau protein, which is conducive to onset of $\mathrm{AD}$ (8). However, the function loss of the NLRP3 inflammasome can reduce 
the hyperphosphorylation and aggregation of tau by regulating tau kinase and phosphorylase. This confirmed the role of the activation of the NLRP3 inflammasome in microglia for the pathogenesis of tau related diseases, and supported the $\mathrm{A} \beta$ cascade hypothesis in the pathogenesis of $\mathrm{AD}$ (49). In addition, the role of neurofibrillary tangles in the downstream development of $\mathrm{A} \beta$-induced microglia activation was also demonstrated (Figure 3).

NLRP3 inflammasome can be activated by a variety of dangerous signals, including pathogenic microorganisms and metabolic disorders. Several studies revealed that in the brains of $\mathrm{AD}$ patients, neuroinflammatory plaques can secrete neurotoxic factors, recruit microglia that engulf the aggregate $A \beta$, and secrete chemically pro-inflammatory molecules, thereby further promoting the damage of surrounding tissues and enhancing the neurotoxic effects of $A \beta$ (50). The deposition of $A \beta$ and the activation of the NLRP3 inflammasome may be mutually causal. The activated intracellular NLRP3 inflammasome can induce M1 phenotype activation of microglia and result in deposition of $\mathrm{A} \beta$, and increased cognitive

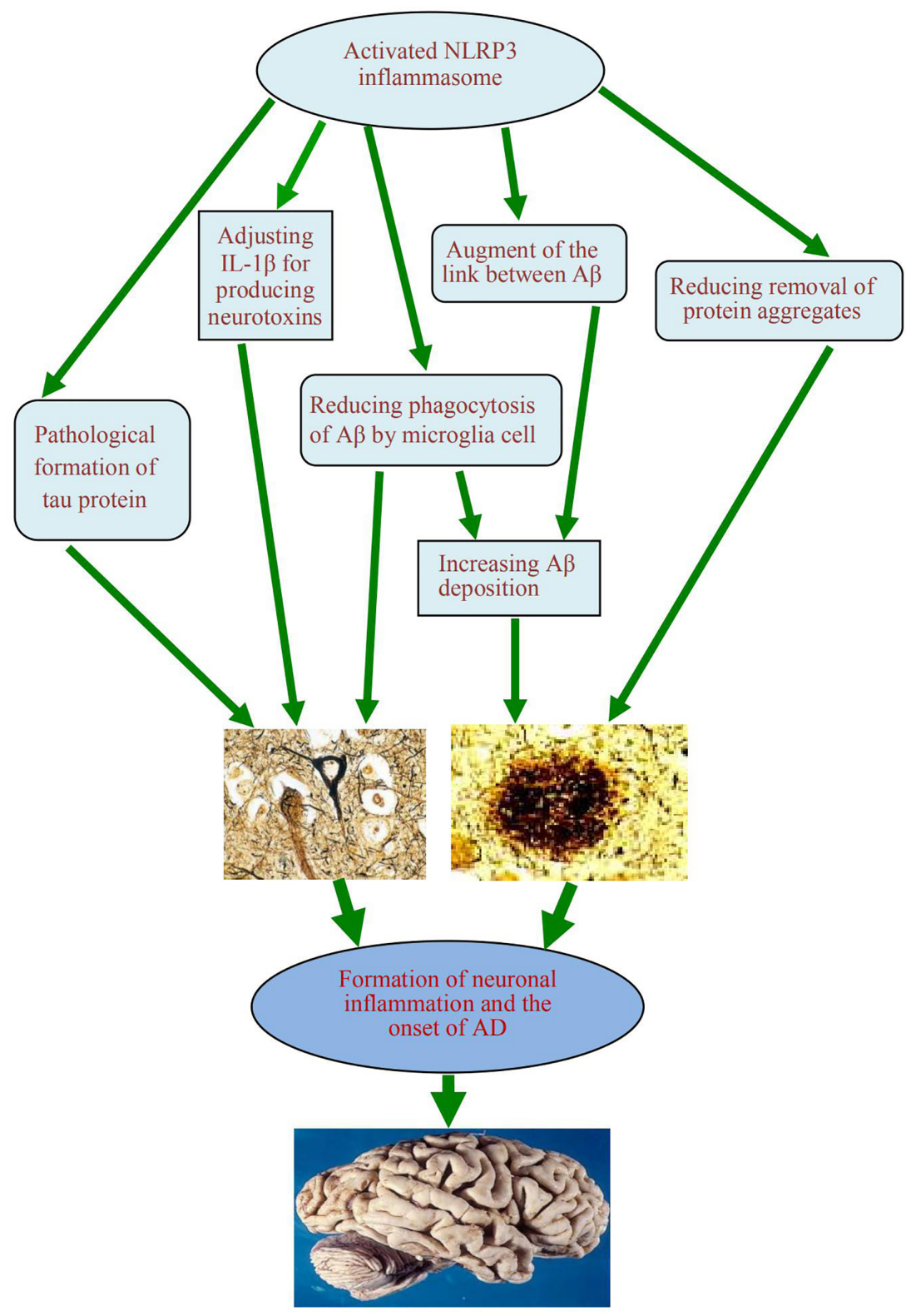

FIGURE 3 | A schematic diagram of the association between activation of NLRP3 inflammasome and occurrence of AD. The activated NLRP3 inflammasome contributes to the formation of chronic neuroinflammation and the pathogenesis of Alzheimer's disease, which play the role by regulating the production of neurotoxin $\mathrm{IL}-1 \beta$, reducing the phagocytosis of $A \beta$ from microglia, augmenting the link between $A \beta$ and $A \beta$, reducing the clearance of some excess protein aggregates, and promoting the pathological formation of Tau protein. NLRP3, nucleotide-binding domain leucine-rich repeat and pyrin domain containing receptor protein 3; AD, Alzheimer's disease; IL-1 $\beta$, interleukin $1 \beta ; A \beta$, $\beta$-amyloid protein. 
impairment in $\mathrm{AD}$ mouse models (21). In contrast, in microglia with specific functional impairment of NLRP3 inflammasome, these cells are biased toward the M2 phenotype, thereby reducing extracellular $\mathrm{A} \beta$ deposition, protecting nerve cells from synaptic dysfunction, and alleviating cognitive decline in the brain (51).

Thioredoxin interaction protein (TXNIP) is an endogenous regulator of REDOX/glucose-induced stress and inflammation. The components of TXNIP and NLRP3 were analyzed by Western Blot, real-time PCR and immunohistochemistry in the cerebral cortex sample from human autopsy, showing that TXNIP protein and mRNA in the cortex of $\mathrm{AD}$ were significantly upregulated. Using double immunofluorescence staining, TXNIP and IL- $1 \beta$ were co-located in the vicinity of $A \beta$ plaque and $p$-tau protein. These results suggest a strong association between TXNIP overexpression and the pathogenesis of $\mathrm{AD}$. It is speculated that the activation of NLRP3 inflammasome in human AD brain may promote the occurrence of neurodegeneration. TXNIP has potential as a molecular link between chronic immune inflammation and $\mathrm{AD}$, and is expected to be a new therapeutic target in the future $(52,53)$.

In fact, the elevated IL-1 $\beta$ level in the brain of patients with $\mathrm{AD}$ and Lewy body pathologies had been reported as early as ten years ago, it may be the end product of some inflammasome activation $(54,55)$. After treating microglia cells with $A \beta$, the expression of IL$1 \beta$ was significantly decreased in the treatment group with lysosomal inhibitor cytochalasin D or cathepsin B inhibitor (46). It is suggested that $A \beta$ can activate the NLRP3 inflammasome by lysosomal rupture pathway to induce the high expression of IL-1 $\beta$, thus promoting the occurrence and development of $\mathrm{AD}$. After their phagocytosis, A $\beta$ fibrils are localized in intracellular lysosomes, and damage to the membranes of these lysosomes results in the release of cathepsin B into the cytoplasmic sol. A pattern recognition receptor cluster of differentiation 36 (CD36) mediated endocytosis pathway that coordinates the transformation of these soluble ligands into crystals or fibers in cells, leading to lysosomal destruction and activation of the NLRP3 inflammasome (38). It should be pointed out that the activation of NLRP3 inflammasome is not always harmful. At least in the early stage, the activation of NLRP3 inflammasome in some tissue cells may help to reduce the damage, or the activation of such inflammasome may have a protective effect on the organism (56).

In order to evaluate the role of NLRP3 inflammasome in the pathogenesis of AD, Heneka et al. (57) used NLRP3 gene knockout mice to hybridize with APP/PS1 transgenic mice. The results showed that there was a lack of caspase cleavage in the brains of the hybrid mice, and that the total IL- $1 \beta$ level was similar to that of wild-type mice, suggesting NLRP3 is important for the pathogenesis of $\mathrm{AD}$. One of the components of the NLRP3 inflammasome, apoptosis-associated speck-like protein containing CARD, was detected by immunohistochemistry. Spot formation of microglia was detected in activated microglia from transgenic APP/PS1 mice with positive Ibal marker, a change consistent with activation of inflammasomes. Wild-type mice, NLRP3 knockout mice, AD mice, and APP/PS1/NLRP3 hybrid knockout mice were tested and compared by water maze test. The results showed that APP/PS1 mice showed severe spatial memory formation defects, while APP/ PS1/NLRP3 hybrid knockout mice showed reduction of spatial and memory impairment to a large extent. By examining the plasticity of synapses in the hippocampus of mice by detecting long-term potentiation (LTP), the authors also found that mice with defects in NLRP3 or caspase-1 completely blocked LTP inhibition (29). As memory is encoded by the plasticity of synapses, LTP is considered to be positively correlated with memory and inversely correlated with the severity of $\mathrm{AD}$. The above experiments confirmed from another perspective that the activation of the NLRP3 inflammasome can promote the onset of $\mathrm{AD}$.

\section{CONTROL OF THE ONSET OF AD BY INHIBITING THE ACTIVATION OF NLRP3 INFLAMMASOME}

Inhibiting the activation of NLRP3 inflammasome has a great prospect for the prevention and treatment of $\mathrm{AD}$. $\beta$-hydroxybutyrate $(\mathrm{BHB})$ is a ketone which can effectively cross the blood-brain barrier and inhibit the activation of NLRP3 inflammasome in human monocytes (58). Some experiments have found that the level of $\mathrm{BHB}$ in the blood of $\mathrm{AD}$ patients is obviously low (59). When the level of $\mathrm{BHB}$ in the blood is increased, the cognitive function of $\mathrm{AD}$ patients is improved (60). Shippy et al. found (61) that the level of BHB in brain tissue and red blood cell samples of $\mathrm{AD}$ patients was significantly lower than that of non ad control group; $\mathrm{BHB}$ inhibited NLRP3 inflammasome in bone marrow-derived macrophages (BMDM) by reducing the levels of IL-1 $\beta$ and caspase-1. 5xFAD mice which is an APP/PS1 transgenic AD mouse model with 5 family gene mutations was used here, $\mathrm{BHB}$ treatment showed significantly reducing the number and volume of pathological plaques in the cerebral cortex of $\mathrm{AD}$ mice. Consistent with the reduction of NLRP3 inflammasome activation, the apoptosis related spot like protein containing caspase recruitment domain in $\mathrm{BHB}$ treated $5 \mathrm{xFAD}$ mice was significantly reduced. It suggests that $\mathrm{BHB}$ or $\mathrm{BH}$ derivatives are promising drugs for the prevention and treatment of $\mathrm{AD}$ (61).

More and more evidence shows that neuroinflammation is an important factor in the onset of Alzheimer's disease (AD). IL-1 $\beta$ may be the main inducer of this inflammation. IL-1 $\beta$ expression is upregulated in the brain of $\mathrm{AD}$ patients, and inhibition of the activation of NLRP3 inflammasome can improve the behavioral abnormalities and synaptic pathological phenotype in $\mathrm{AD}$ mice (39). Dapansutrile (OLT1177) is a specific inhibitor of NLRP3. Lonnemann et al. (62) used OLT1177 to inhibit the activation of NLRP3 inflammasome and thus improve the function of AD mice. Their experiment showed: 6 months when the size of APP/PS1 mice were fed standard chow and add OLT1177 food after 3 months, can improve the learning and memory ability of mice, normalize $\mathrm{AD}$ metabolic markers in plasma, and restore the synaptic plasticity, reduce the number of pathological plaques in the cerebral cortex, and reducing the brain glial cell activity. In the future, OLT1177 is expected to be developed as a new drug for the treatment of $\mathrm{AD}$. Diacetyl-p-phenylenediamine is a synthetic chemical containing benzene ring, which has the ability to regulate the function of microglia, inhibit neuronal inflammation, and promote the elimination and alleviation of cognitive impairment in $\mathrm{AD}$ brain. 
In transgenic $\mathrm{AD}$ mouse model, diacetyl-p-phenylenediamine can promote microglia phagocytosis and improve the cognitive function of mice by affecting NF- $\mathrm{KB}$ signaling pathway and inhibiting the expression of NLRP3 Dysfunction (63).

Some herbs or their extract can inhibit the activation of NLRP3 inflammasome and show the effect of prevention and treatment of AD (64-66). Radix Scrophulariae (RS) is a kind of herb that has anti-oxidation, anti-inflammatory, anti-allergic, and anti-cancer features. Kim et al. (67) found that IL-1 $\beta$ was effectively reduced after treatment with RS, along with the downregulation of NLRP3 protein, and inhibition of caspase- 1 activity in 5XFAD mice. Two months after treatment with RS, A $\beta$ and plaque bulk were obviously decreased in the hippocampus of the brains of 5XFAD mice after treating with Radix Scrophulariae. In addition, RS can regulate neuroinflammation and down-regulate the expression of BACE1 protein in 5XFAD mice. BACE1 is a protein that can increase $A \beta$ formation. It has been found that fresh coconut oil shows potential neuroprotective effects by inhibiting the activation of NLRP3 inflammasomes, mainly by antagonizing $A \beta$-induced neurotoxicity (68). Pterostilbene is a compound extracted from grapes and strawberries and can inhibits $A \beta$-induced neuroinflammation in a microglia cell line by inactivating the NLRP3 inflammasome (69).

Neuroinflammation and autophagy dysfunction are involved in the pathological process of $\mathrm{AD}$, and the autophagy-lysosomal pathway is a specific molecular mechanism that modulates $A \beta$ induced activation of NLRP3-caspase-1 inflammasome. Hong et al. found that progesterone (PG) exhibited a neuroprotective effect by inhibiting $A \beta$-induced activation of NLRP3 inflammasomes by enhancing autophagy in astrocytes (70). Stavudine (D4T) is a nucleoside reverse transcriptase inhibitor, which can reduce NLRP3 assembly as well as IL-18 and caspase-1 production, but did not affect IL-1 $\beta$ production and TREM2 expression. In an in vitro $\mathrm{AD}$ model of $\mathrm{A} \beta$-driven neuroinflammation, $\mathrm{D} 4 \mathrm{~T}$ reduces NLRP3 inflammasome-associated inflammation and stimulates $\mathrm{A} \beta$ autophagy by macrophages (58). 2,3,5,4 '-Tetrahydroxy stilbene-2$\mathrm{O}-\beta$-D-glucoside (TSG) is a promising chemical for drug development in the treatment of $\mathrm{AD}$, which has been shown to be beneficial in reducing cognitive function in animal models of AD. TSG can effectively alleviate LPS-induced neuroinflammatory response by inhibiting the NLRP3 signaling pathway of microglia and neurons. Meanwhile, it can inhibit the activity of NLRP3 inflammasome and regulate mitochondrial autophagy, especially in microglia cells. In addition, TSG also promoted autophagy involved in AMPK/PINK1/Parkin signaling pathway, suggesting a neuroprotective effect dependent on PINK1. When PINK1 is deficient, autophagy is inhibited. Knocking out PINK1 or Parkin genes via the CRISPR/Cas9 system may disrupt the neuroprotective effects of TSG (71).

\section{REFERENCES}

1. Honig LS, Vellas B, Woodward M, Boada M, Bullock R, Borrie M, et al. Trial of Solanezumab for Mild Dementia Due to Alzheimer's Disease. N Engl J Med (2018) 378(4):321-30. doi: 10.1056/NEJMoa1705971

\section{CONCLUSIONS}

Chronic inflammation during $\mathrm{AD}$ is mainly mediated by abnormal immune function of brain cells, which is generally believed to be caused by the abnormal accumulation of some proteins in microglia, mainly related to the activation of NLRP3 inflammasomes (72). As for the pathogenesis of $\mathrm{AD}$, there have been the theory of $\beta$-amyloid protein, the theory of abnormal neurotransmitters, the theory of abnormal Tau protein metabolism and the theory of nerve cell apoptosis. Recently, the antimicrobial protection theory of $\mathrm{AD}$ disease has attracted attention. This theory holds that $A \beta$ is a highly conserved innate immune effector molecule, and the oligomerization of $A \beta$ protein and the production of $\beta$-amyloid are important innate immune pathways that mediate pathogen capture and protect infection. The $\beta$-amyloid deposition mimics an innate immune response to perceived immune stimulation, and since the brains of some $\mathrm{AD}$-susceptible patients may sometimes have microbial infections, $A \beta$ first traps and neutralizes invading pathogens with $\beta$-amyloid, acting as an antimicrobial peptide-like protein (73). A $\beta$ fibrosis drives neuroinflammatory pathways, helps fight infection and clears deposition of harmful pathogens. In the pathogenesis of $\mathrm{AD}$, the abnormal pathophysiological behavior pattern of $A \beta$ is manifested as an innate immune response disorder, leading to persistent chronic immune inflammation and neurodegenerative diseases (74). Theory of neuroinflammation induced by activation of NLRP3 inflammasome suggests that congenital immune disorders and neuroinflammation play an important role in the pathogenesis of AD (75). NLRP3 inflammasome activators promote neuroinflammation and senile plaque formation through a variety of pathways. NLRP3 inflammasomes usually remains activation and regulate the excretion of harmful inflammatory molecules in the brains of $\mathrm{AD}$ patients. Inhibition of the activation of NLRP3 inflammasome may be an important way to treat $\mathrm{AD}$ patients in the future.

\section{AUTHOR CONTRIBUTIONS}

HB designed and wrote the manuscript. QZ discussed and revised the manuscript. All authors contributed to the article and approved the submitted version.

\section{FUNDING}

This work was supported by National Natural Science Foundation of China (to BH), No:32060182, and the grant of Science and Technology Support Plan of Guizhou Province in China, No. QianKeHe-Zhicheng [2020]4Y129 (to BH).

2. Yamazaki Y, Zhao N, Caulfield TR, Liu CC, Bu G. Apolipoprotein E and Alzheimer Disease: Pathobiology and Targeting Strategies. Nat Rev Neurol (2019) 15(9):501-18. doi: 10.1038/s41582-019-0228-7

3. Querfurth HW, LaFerla FM. Alzheimer's Disease. N Engl J Med (2010) 362 (4):329-44. doi: 10.1056/NEJMra0909142 
4. Falcon B, Zivanov J, Zhang W, Murzin AG, Garringr HJ, Vidal R, et al. Novel Tau Filament Fold in Chronic Traumatic Encephalopathy Encloses Hydrophobic Molecules. Nature (2019) 568(7752):420-3. doi: 10.1038/s41586-019-1026-5

5. Hardy J, Selkoe DJ. The Amyloid Hypothesis of Alzheimer's Disease: Progress and Problems on the Road to Therapeutics. Science (2002) 297(5580):353-6. doi: $10.1126 /$ science. 1072994

6. Mathys H, Davila-Velderrain J, Peng Z, Gao F, Mohammadi S, Young JZ, et al. Single-Cell Transcriptomic Analysis of Alzheimer's Disease. Nature (2019) 570(7761):332-7. doi: 10.1038/s41586-019-1195-2

7. Kim SM, Ha JS, Han AR, Cho SW, Yang SJ. Effects of $\alpha$-Lipoic Acid on LPSInduced Neuroinflammation and NLRP3 Inflammasome Activation Through the Regulation of BV-2 Microglial Cells Activation. BMB Rep (2019) 52(10):613-8. doi: 10.5483/BMBRep.2019.52.10.026

8. Ising C, Venegas C, Zhang S, Scheiblich H, Schmidt SV, Vieira-Saecker A, et al. NLRP3 Inflammasome Activation Drives Tau Pathology. Nature (2019) 575(7784):669-73. doi: 10.1038/s41586-019-1769-z

9. Wu PJ, Hung YF, Liu HY, Hsueh YP. Deletion of the Inflammasome Sensor Aim2 Mitigates A $\beta$ Deposition and Microglial Activation But Increases Inflammatory Cytokine Expression in an Alzheimer Disease Mouse Model. Neuroimmunomodulation (2017) 24(1):29-39. doi: 10.1159/000477092

10. Vajjhala PR, Mirams RE, Hill JM. Multiple Binding Sites on the Pyrin Domain of ASC Protein Allow Self-Association and Interaction With NLRP3 Protein. J Biol Chem (2012) 287:41732-43. doi: 10.1074/jbc.M112.381228

11. Zeng CY, Li CG, Shu JX, Xu LH, Ouyang DY, Mai FY, et al. ATP Induces Caspase-3/Gasdermin E-Mediated Pyroptosis in NLRP3 Pathway-Blocked Murine Macrophages. Apoptosis (2019) 24(9-10):703-17. doi: 10.1007/ s10495-019-01551-x

12. Pellegrini C, Fornai M, Antonioli L, Blandizzi C, Calderone V. Hytochemicals as Novel Therapeutic Strategies For NLRP3 Inflammasome-Related Neurological, Metabolic, and Inflammatory Diseases. Int J Mol Mol Sci (2019) 20(12):2876. doi: 10.3390/ijms20122876

13. Lin KM, Hu W, Troutman TD, Jennings M, Brewer T, Li X, et al. IRAK-1 Bypasses Priming and Directly Links TLRs to Rapid NLRP3 Inflammasome Activation. Proc Natl Acad Sci USA (2014) 111:775-80. doi: 10.1073/ pnas. 1320294111

14. Lamkanfifi M, Dixit VM. Mechanisms and Functions of Inflammasomes. Cell (2014) 157:1013-22. doi: 10.1016/j.cell.2014.04.007

15. Narayanan KB, Park HH. Purification and Analysis of the Interactions of Caspase-1 and ASC for Assembly of the Inflammasome. Appl Biochem Biotechnol (2015) 175(6):2883-94. doi: 10.1007/s12010-014-1471-4

16. Youm YH, Nguyen KY, Grant RW, Goldberg EL, Bodogai M, Kim D, et al. The Ketone Metabolite $\beta$-Hydroxybutyrate Blocks NLRP3 InflammasomeMediated Inflammatory Disease. Nat Med (2015) 21(3):263-9. doi: 10.1038/ nm.3804

17. Aftabizadeh M, Tatarek-Nossol M, Andreetto E, El Bounkari O, Kipp M, Beyer C, et al. Blocking Inflammasome Activation Caused by $\beta$-Amyloid Peptide (A $\beta$ ) and Islet Amyloid Polypeptide (IAPP) Through an IAPP Mimic. ACS Chem Neurosci (2019) 10(8):3703-17. doi: 10.1021/acschemneuro.9b00260

18. Franchi L, Eigenbrod T, Muñoz-Planillo R, Nuñez G. The Inflammasome: a Caspase-1 Activation Platform That Regulates Immune Responses and Disease Pathogenesis. Nat Immunol (2009) 10(3):241-7. doi: 10.1038/ni.1703

19. Gu Y, Wu J, Faucheu C, Lalanne JL, Diu A, Livingston DJ, et al. Interleukin-1 Beta Converting Enzyme Requires Oligomerization for Activity of Processed Forms In Vivo. EMBO J (1995) 14(9):1923-31. doi: 10.1002/j.14602075.1995.tb07184.x

20. Aricioglu F, Ozkartal CS, Bastaskin T, Tüzün E, Kandemir C, Sirvanci S, et al. Antidepressant-Like Effects Induced by Chronic Blockade of the Purinergic 2x7 Receptor Through Inhibition of Non-Like Receptor Protein 1 Inflammasome in Chronic Unpredictable Mild Stress Model of Depression in Rats. Clin Psychopharmacol Neurosci (2019) 17(2):261-72. doi: 10.9758/ cpn.2019.17.2.261

21. Cherry JD, Olschowka JA, O'Banion MK. Neuroinflammation and M2 Microglia: The Good, the Bad, and the Inflamed. J Neuroinflammat (2014) 11(1):98-106. doi: 10.1186/1742-2094-11-98

22. Lawana V, Singh N, Sarkar S, Charli A, Jin H, Anantharam V, et al. Involvement of C-Abl Kinase in Microglial Activation Of NLRP3 Inflammasome and Impairment in Autolysosomal System. Neuroimmune Pharmacol (2017) 12 (4):624-60. doi: 10.1007/s11481-017-9746-5
23. Zhang Y, Wang L, Lv Y, Jiang C, Hu G. The GTPase Rab1 Is Required for NLRP3 Inflammasome Activation and Inflammatory Lung Injury. J Immunol (2019) 202(1):194-206. doi: 10.4049/jimmunol.1800777

24. Zhou R, Tardivel A, Thorens B, Choi I, Tschopp J. Thioredoxin-Interacting Protein Links Oxidative Stress to Inflammasome Activation. Nat Immunol (2010) 11(2):136-40. doi: 10.1038/ni.1831

25. Lee GS, Subramanian N, Kim AI, Aksentijevich I, Goldbach-Mansky R, Sacks $\mathrm{DB}$, et al. The Calcium-Sensing Receptor Regulates the NLRP3 Inflammasome Through Ca2+ and cAMP. Nature (2012) 492(7427):123-7. doi: 10.1038/ nature11588

26. Savage CD, Lopez-Castejon G, Denes A, Brough D. NLRP3-Inflammasome Activating DAMPs Stimulate an Inflammatory Response in Glia in the Absence of Priming Which Contributes to Brain Inflammation After Injury. Front Immunol (2012) 3:288. doi: 10.3389/fimmu.2012.00288

27. Suzuki T, Kohyama K, Moriyama K, Ozaki M, Hasegawa S, Ueno T, et al. Extracellular ADP Augments Microglial Inflammasome and NF- $\mathrm{kb}$ Activation via the P2Y12 Receptor. Eur J Immunol (2020) 50(2):205-19. doi: $10.1002 /$ eji.201848013

28. Wyss-Coray T. Inflammation in Alzheimer Disease: Driving Force, Bystander or Beneficial Response? Nat Med (2006) 12:1005-15. doi: 10.1038/nm1484

29. Murphy N, Cowley TR, Richardson JC, Virley D, Upton N, Walter D, et al. The Neuroprotective Effect of a Specific P2X7 Receptor Antagonist Derives From its Ability to Inhibit Assembly of the NLRP3 Inflammasome in Glial Cells. Brain Pathol (2012) 22(3):295-306. doi: 10.1111/j.17503639.2011.00531.x

30. Mahmoudiasl GR, Abbaszadeh HA, Rezaei-Tavirani M, Abdollahifar MA, Khoramgah MS, Niknazar S, et al. Nod-Like Receptor Protein 3 and Nod-Like Receptor Protein 1 Inflammasome Activation in the Hippocampal Region of Postmortem Methamphetamine Chronic User. Bratisl Lek Listy (2019) 120 (10):769-76. doi: 10.4149/BLL_2019_129

31. Chen X, Liu G, Yuan Y, Wu G, Wang S, Yuan L. NEK7 interacts With NLRP3 to Modulate the Pyroptosis in Inflammatory Bowel Disease Via NF-kb Signaling. Cell Death Dis (2019) 10(12):906. doi: 10.1038/s41419019-2157-1

32. Sharif H, Wang L, Wang WL, Magupalli VG, Andreeva L, Qiao Q, et al. Structural Mechanism for NEK7-Licensed Activation of NLRP3 inflammasome. Nature (2019) 570(7761):338-43. doi: 10.1038/s41586-019-1295-Z

33. Ren G, Zhang X, Xiao Y, Zhang W, Wang Y, Ma W, et al. ABRO1 Promotes NLRP3 Inflammasome Activation Through Regulation of NLRP3 Deubiquitination. EMBO J (2019) 386:e100376. doi: 10.15252/embj.2018100376

34. Katsnelson MA, Rucker LG, Russo HM, Dubyak GR. K+ Efflux Agonists Induce NLRP3 Inflammasome Activation Independently of Ca2+ Signaling. J Immunol (2015) 194(8):3937-52. doi: 10.4049/jimmunol.1402658

35. Zhang Y, Rong H, Zhang FX, Wu K, Mu L, Meng J, et al. A Membrane Potentialand Calpain-Dependent Reversal of Caspase-1 Inhibition Regulates Canonical NLRP3 Inflammasome. Cell Rep (2018) 24(9):2356-69. doi: 10.1016/ j.celrep.2018.07.098

36. Wu AG, Zhou XG, Qiao G, Yu L, Tang Y, Yang L, et al. Targeting Microglial Autophagic Degradation in NLRP3 Inflammasome-Mediated Neurodegenerative Diseases. Ageing Res Rev (2021) 65:101202. doi: 10.1016/ j.arr.2020.101202

37. Bai H, Zhang Q, Duan J, Yu DJ, Liu LJ. Downregulation of Signal Transduction and STAT3 Expression Exacerbate the Oxidative Stress Mediated by NLRP3 Inflammasome. Neural Regeneration Res (2018) 13 (12):2147-55. doi: 10.4103/1673-5374.241470

38. Sharma D, Kanneganti TD. The Cell Biology of Inflammasomes: Mechanisms of Inflammasome Activation and Regulation. J Cell Biol (2016) 213:617-29. doi: $10.1083 /$ jcb.201602089

39. Gold M, El Khoury J. Beta-Amyloid, Microglia, and the Inflammasome in Alzheimer's Disease. Semin Immuno Pathol (2015) 37:607-11. doi: 10.1007/ s00281-015-0518-0

40. Shimada K, Crother TR, Karlin J, Dagvadorj J, Chiba N, Chen S, et al. Oxidized Mitochondrial DNA Activates the NLRP3 in Flammasome During Apotosis. Immunity (2012) 36(3):401-14. doi: 10.1016/j.immuni. 2012.01.009

41. Gong Z, Pan J, Shen Q, Li M, Peng Y. Mitochondrial Dysfunction Induces NLRP3 inflammasome Activation During Cerebral Ischemia/Reperfusion Injury. J Neuroinflamm (2018) 15(1):242. doi: 10.1186/s12974-018-1282-6 
42. Chokchaiwong S, Kuo YT, Lin SH, Hsu YC, Hsu SP, Kao SH. Coenzyme Q10 Serves to Couple Mitochondrial Oxidative Phosphorylation and Fatty Acid $\beta$ Oxidation, and Attenuates NLRP3 inflammasome Activation. Free Radic Res (2018) 52(11-12):1445-55. doi: 10.1080/10715762.2018.1500695

43. Alehashemi S, Goldbach-Mansky R. Human Autoinflammatory Diseases Mediated by NLRP3-, Pyrin-, NLRP1-, and NLRC4-Inflammasome Dysregulation Updates on Diagnosis, Treatment, and the Respective Roles of IL-1 and IL-18. Front Immunol (2020) 11:1840. doi: 10.3389/fimmu.2020.01840

44. Friker LL, Scheiblich H, Hochheiser IV, Brinkschulte R, Riedel D, Latz E, et al. $\beta$-Amyloid Clustering Around ASC Fibrils Boosts Its Toxicity in Microglia. Cell Rep (2020) 30(11):3743-54. doi: 10.1016/j.celrep.2020.02.025

45. Furman D, Chang J, Lartigue L. Expression of Specific Inflammasome Gene Modules Stratifies Older Individuals Into Two Extreme Clinical and Immunological States. Nat Med (2017) 23(2):174-84. doi: 10.1038/nm.4267

46. Lučiūnaitè A, McManus RM, Jankunec M, Rácz I, Dansokho C, Dalgèdienè I, et al. Soluble $A \beta$ Oligomers and Protofibrils Induce NLRP3 Inflammasome Activation in Microglia. J Neurochem (2019) 23:e14945. doi: 10.1111/jnc.14945

47. Ahmed ME, Iyer S, Thangavel R, Kempuraj D, Selvakumar GP, Raikwar SP, et al. Co-Localization of Glia Maturation Factor With NLRP3 Inflammasome and Autophagosome Markers in Human Alzheimer's Disease Brain. J Alzheimers Dis (2017) 60(3):1143-60. doi: 10.3233/JAD-170634

48. Saresella M, La Rosa F, Piancone F, Zoppis M, Marventano I, Calabrese E, et al. The NLRP3 and NLRP1 Inflammasomes Are Activated in Alzheimer's Disease. Mol Neurodegener (2016) 11(1):23-37. doi: 10.1186/s13024-016-0088-1

49. Dempsey C, Rubio Araiz A, Bryson KJ, Finucane O, Larkin C, Mills EL, et al. Inhibiting the NLRP3 Inflammasome With MCC950 Promotes nonPhlogistic Clearance of Amyloid- $\beta$ and Cognitive Function in APP/PS1 Mice. Brain Behav Immun (2017) 61:306-16. doi: 10.1016/j.bbi.2016.12.014

50. Tschopp J, Schroder K. NLRP3 Inflammasome Activation: The Convergence of Multiple Signalling Pathways on ROS Production? Nat Rev Immunol (2010) 10(3):210-5. doi: 10.1038/nri2725

51. Zhou R, Yazdi AS, Menu P, Tschopp J. A Role for Mitochondria in NLRP3 Inflammasome Activation. Nature (2011) 469(7329):221-5. doi: 10.1038/ nature 09663

52. Li L, Ismael S, Nasoohi S, Sakata K, Liao FF, McDonald MP, et al. ThioredoxinInteracting Protein (TXNIP) Associated NLRP3 Inflammasome Activation in Human Alzheimer's Disease Brain. J Alzheimers Dis (2019) 68(1):255-65. doi: 10.3233/JAD-180814

53. Tsubaki H, Tooyama I, Walker DG. Thioredoxin-Interacting Protein (TXNIP) With Focus on Brain and Neurodegenerative Diseases. Int J Mol Sci (2020) 21(24):9357. doi: 10.3390/ijs21249357

54. Panicker N, Sarkar S, Harischandra DS, Neal M, Kam TI, Jin H, et al. Fyn Kinase Regulates Misfolded Alpha-Synuclein Uptake and NLRP3 inflammasome activation in Microglia. J Exp Med (2019) 216(6):1411-30. doi: $10.1084 /$ jem.20182191

55. Lamkanfi M, Dixit V. Inflammasomes: Guardians of Cytosolic Sanctity. Immunol Rev (2009) 227(1):95-105. doi: 10.1111/j.1600-065X.2008.00730.x

56. Sayed RKA, Fernández-Ortiz M, Diaz-Casado ME, Aranda-Martínez $P$, Fernández-Martínez J, Guerra-Librero A, et al. Lack of NLRP3 Inflammasome Activation Reduces Age-Dependent Sarcopenia and Mitochondrial Dysfunction, Favoring the Prophylactic Effect of Melatonin. Biol Sci Med Sci (2019) 74(11):1699-708. doi: 10.1093/gerona/glz079

57. Heneka MT, Kummer MP, Stutz A, Delekate A, Schwartz S, Vieira-Saecker A, et al. NLRP3 Is Activated in Alzheimer's Disease and Contributes to Pathology in APP/PS1 Mice. Nature (2013) 493(7434):674-8. doi: 10.1038/nature11729

58. La Rosa F, Saresella M, Marventano I, Piancone F, Ripamonti E, Al-Daghri N, et al. Stavudine Reduces NLRP3 Inflammasome Activation and Modulates Amyloid- $\beta$ Autophagy. J Alzheimers Dis (2019) 72(2):401-12. doi: 10.3233/ JAD-181259

59. Ota M, Matsuo J, Ishida I, Takano H, Yokoi Y, Hori H, et al. Effects of a Medium-Chain Triglyceride-Based Ketogenic Formula on Cognitive Function in Patients With Mild-to-Moderate Alzheimer's Disease. Neurosci Lett (2019) 690:232-6. doi: 10.1016/j.neulet.2018.10.048

60. Reger MA, Henderson ST, Hale C, Cholerton B, Baker LD, Watson GS, et al. Effects of Beta-Hydroxybutyrate on Cognition in Memory-Impaired Adults. Neurobiol Aging (2004) 25:311-4. doi: 10.1016/S0197-4580(03)00087-3

61. Shippy DC, Wilhelm C, Viharkumar PA, Raife TJ, Ulland TK. $\beta$ Hydroxybutyrate Inhibits Inflammasome Activation to Attenuate
Alzheimer's Disease Pathology. J Neuroinflamm (2020) 17(1):280. doi: 10.1186/s12974-020-01948-5

62. Lonnemann N, Hosseini S, Marchetti C, Skouras DB, Stefanoni D, D'Alessandro A, et al. The NLRP3 Inflammasome Inhibitor OLT1177 Rescues Cognitive Impairment in a Mouse Model of Alzheimer's Disease. Proc Natl Acad Sci USA (2020) 117(50):32145-54. doi: 10.1073/pnas.2009680117

63. Park MH, Lee M, Nam G, Kim M, Kang J, Choi BJ, et al. N,N'-Diacetyl-PPhenylenediamine Restores Microglial Phagocytosis and Improves Cognitive Defects in Alzheimer's Disease Transgenic Mice. Proc Natl Acad Sci USA (2019) 116(47):23426-36. doi: 10.1073/pnas.1916318116

64. Qiu WQ, Pan R, Tang Y, Zhou XG, Wu JM, Yu L, et al. Lychee Seed Polyphenol Inhibits Abeta- Induced Activation of NLRP3 Inflammasome via the LRP1/AMPK Mediated Autophagy Induction. BioMed Pharmacother (2020) 130:110575. doi: 10.1016/j.biopha.2020.110575

65. Chen DB, Gao HW, Peng C, Pei SQ, Dai AR, Yu XT, et al. Quinones as Preventive Agents in Alzheimer's Diseases: Focus on NLRP3 inflammasomes. J Pharm Pharmacol (2020) 72(11):1481-90. doi: 10.1111/jphp.13332

66. Wang CY, Xu Y, Wang X, Guo C, Wang T, Wang ZY. Dl-3-N-Butylphthalide Inhibits NLRP3 Inflammasome and Mitigates Alzheimer's-Like Pathology via Nrf2-TXNIP -TrX Axis. Antioxid Redox Signal (2019) 30(11):1411-31. doi: 10.1089/ars.2017.7440

67. Kim N, Do J, Ju IG, Jeon SH, Lee JK, Oh MS. Picrorhiza Kurroa Prevents Memory Deficits by Inhibiting NLRP3 Inflammasome Activation and BACE1 Expression in 5xfad Mice. Neurotherapeutics (2020) Jan17(1):189-99. doi: 10.1007/s13311-019-00792-7

68. Mirzaei F, Khazaei M, Komaki A, Amiri I, Jalili C. Virgin Coconut Oil (VCO) by Normalizing NLRP3 Inflammasome Showed Potential Neuroprotective Effects in Amyloid-Beta Induced Toxicity and High-Fat Diet Fed Rat. Food Chem Toxicol (2018) 118:68-83. doi: 10.1016/j.fct.2018.04.064

69. Li Q, Chen L, Liu X, Li X, Cao Y, Bai Y, et al. Pterostilbene Inhibits AmyloidBeta-Induced Neuroinflammation in a Microglia Cell Line by Inactivating the NLRP3/caspase-1 Inflammasome Pathway. J Cell Biochem (2018) 119:705362. doi: $10.1002 / j c b .27023$

70. Hong Y, Liu Y, Yu D, Zhang L, Pi J, Sun H, et al. The Neuroprotection of Progesterone Against A $\beta$-Induced NLRP3-Caspase-1 Inflammasomeactivation via Enhancing Autophagy in Astrocytes. Int Immunopharmacol (2019) 74:105669. doi: 10.1016/j.intimp.2019.05.054

71. Gao Y, Li J, Li J, Hu C, Zhang L, Yang J, et al. Tetrahydroxy Stilbene Glycoside Alleviated Inflammatory Damage by Mitophagy via AMPK Related PINK1/ Parkin Signaling Pathway. Biochem Pharmacol (2020) 177:113997. doi: 10.1016/j.bcp.2020.113997

72. Abbott A. Is 'Friendly Fire' in the Brain Provoking Alzheimer's Disease? Nature (2018) 556(7702):426-8. doi: 10.1038/d41586-018-04930-7

73. Abbott A. Are Infection Seeding Some Cases of Alzheimer's Disease? Nature (2020) 587(7832):22-5. doi: 10.1038/d41586-020-03084-9

74. Moir RD, Lathe R, Tanzi RE. The Antimicrobial Protection Hypothesis of Alzheimer's Disease. Alzheimer's Dementia (2018) 14:1602-14. doi: 10.1016/ j.jalz.2018.06.3040

75. Heppner FL, Ransohoff RM, Becher B. Immune Attack: The Role of Inflammation in Alzheimer Disease. Nat Rev Neurosci (2015) 16(6):35872. doi: $10.1038 / \mathrm{nrn} 3880$

Conflict of Interest: The authors declare that the research was conducted in the absence of any commercial or financial relationships that could be construed as a potential conflict of interest.

Publisher's Note: All claims expressed in this article are solely those of the authors and do not necessarily represent those of their affiliated organizations, or those of the publisher, the editors and the reviewers. Any product that may be evaluated in this article, or claim that may be made by its manufacturer, is not guaranteed or endorsed by the publisher.

Copyright $\odot 2021$ Bai and Zhang. This is an open-access article distributed under the terms of the Creative Commons Attribution License (CC BY). The use, distribution or reproduction in other forums is permitted, provided the original author(s) and the copyright owner(s) are credited and that the original publication in this journal is cited, in accordance with accepted academic practice. No use, distribution or reproduction is permitted which does not comply with these terms. 